\title{
Pilotstudie zur Wirksamkeit einer multimodalen Gruppenbehandlung für Kinder mit einer Störung des Sozialverhaltens mit oppositionellem, aufsässigem Verhalten in der klinischen Grundversorgung
}

\author{
Wettach, Ralph ; Aebi, Marcel
}

\begin{abstract}
Fragestellung: Die Störung des Sozialverhaltens mit oppositionellem, aufsässigem Verhalten (SSV/OAV) ist eine häufige Störung des Kindesalters und oft Zuweisungsgrund an kinderpsychiatrische Kliniken. Die SSV/OAV zeigt sich in Form von erhöhter Reizbarkeit, Dickköpfigkeit und boshaften Verhaltensweisen. In dieser Pilotstudie sollen Hinweise auf die Wirksamkeit von kombinierten kognitivverhaltenstherapeutischen Gruppeninterventionen - bei den Kindern das Baghira-Gruppentraining und bei deren Eltern das Triple P-Elterntraining - im Kontext der ambulanten kinderpsychiatrischen Grundversorgung geprüft werden. Methodik: 42 Kinder mit SSV/OAV erhielten die kombinierte Behandlung und wurden mit 26 unbehandelten Probanden einer Wartelistekontrollgruppe verglichen. Externalisierendes Problemverhalten sowie aggressives und regelverletzendes Verhalten wurden bei Behandlungsbeginn und -ende sowie nach sechs Monaten Katamnesezeitraum mittels der Fragebogenmethode bei Kindern, Eltern und Lehrpersonen erhoben. Ergebnisse: Im Vergleich zur Wartelistekontrollgruppe ergab sich bei den Kindern der Behandlungsgruppe zwischen Prä- und Posterhebung eine signifikante Abnahme der von den Eltern beurteilten externalisierenden Probleme und eine tendenziell signifikante Abnahme des von den Lehrpersonen berichteten regelverletzenden Verhaltens. Es wurden mittelgroße Effektstärken gefunden und die Effekte blieben über den Katamnesezeitraum stabil. Schlussfolgerungen: In dieser Pilotstudie zeigten sich die kombinierten Interventionen für die Behandlung von SSV/OAV in der ambulanten kinderpsychiatrischen Grundversorgung als wirksam und hilfreich. Zur Beurteilung der spezifischen Wirksamkeit des Baghira-Gruppentrainings sind weitere, umfangreichere Studien erforderlich
\end{abstract}

DOI: https://doi.org/10.1024/1422-4917/a000424

Other titles: Pilot study on the effectiveness of a multimodal group treatment for children with oppositional defiant disorder in clinical primary care

Posted at the Zurich Open Repository and Archive, University of Zurich

ZORA URL: https://doi.org/10.5167/uzh-134729

Journal Article

Accepted Version

Originally published at:

Wettach, Ralph; Aebi, Marcel (2016). Pilotstudie zur Wirksamkeit einer multimodalen Gruppenbehandlung für Kinder mit einer Störung des Sozialverhaltens mit oppositionellem, aufsässigem Verhalten in der klinischen Grundversorgung. Zeitschrift für Kinder- und Jugendpsychiatrie und Psychotherapie, 44(3):220-230.

DOI: https://doi.org/10.1024/1422-4917/a000424 


\section{Pilotstudie zur Wirksamkeit einer multimodalen}

\section{Gruppenbehandlung für Kinder mit einer Störung}

des Sozialverhaltens mit oppositionellem,

aufsässigen Verhalten in der klinischen

\section{Grundversorgung}

Ralph Wettach ${ }^{1,2}$ und Marcel Aebi ${ }^{1,3,4}$

${ }^{1}$ Universitätsklinik für Kinder- und Jugendpsychiatrie, Universität Zürich, Schweiz

${ }^{2}$ Schulgesundheitsdienste der Stadt Zürich, Schulpsychologischer Dienst, Schweiz

${ }^{3}$ Psychiatrische Universitätsklinik Zürich, Klinik für Forensische Psychiatrie, Zentrum für Kinder- und Jugendforensik, Schweiz

${ }^{4}$ Psychologisches Institut, Klinische Psychologie Kinder/Jugendliche \& Paare Familien, Universität Zürich, Schweiz 


\section{Zusammenfassung}

Fragestellung: Die Störung des Sozialverhaltens mit oppositionellem, aufsässigen Verhalten (SSV/OAV) ist eine häufige Störung des Kindesalters und oft Zuweisungsgrund an kinderpsychiatrische Kliniken. Die SSV/OAV zeigt sich in Form von erhöhter Reizbarkeit, Dickköpfigkeit und boshaften Verhaltensweisen. In dieser Pilotstudie sollen Hinweise auf die Wirksamkeit von kombinierten kognitivverhaltenstherapeutischen Gruppeninterventionen - bei den Kindern das Baghira-Gruppentraining und bei deren Eltern das Triple $\mathrm{P}$ Elterntraining - im Kontext der ambulanten kinderpsychiatrischen Grundversorgung geprüft werden.

Methodik: 42 Kinder mit SSV/OAV erhielten die kombinierte Behandlung und wurden mit 26 unbehandelten Probanden einer Wartelistekontrollgruppe verglichen. Externalisierendes Problemverhalten sowie aggressives und regelverletzendes Verhalten wurden bei Behandlungsbeginn und -ende sowie nach sechs Monaten Katamnesezeitraum mittels der Fragebogenmethode bei Kindern, Eltern und Lehrpersonen erhoben.

Ergebnisse: Im Vergleich zur Wartelistekontrollgruppe ergab sich bei den Kindern der Behandlungsgruppe zwischen Prä- und PostErhebung eine signifikante Abnahme der von den Eltern beurteilten externalisierenden Probleme und eine tendenziell signifikante Abnahme des von den Lehrpersonen berichteten regelverletzenden Verhaltens. Es wurden mittelgrosse Effektstärken gefunden und die Effekte blieben über den Katamnesezeitraum stabil. 
Schlussfolgerungen: In dieser Pilotstudie zeigten sich die kombinierten Interventionen für die Behandlung von SSV/OAV in der ambulanten kinderpsychiatrischen Grundversorgung als wirksam und hilfreich. Zur Beurteilung der spezifischen Wirksamkeit des BaghiraGruppentrainings sind weitere, umfangreichere Studien erforderlich. Schlüsselbegriffe: Störung des Sozialverhaltens mit oppositionellem aufsässigen Verhalten, kognitive Verhaltenstherapie, Gruppentherapie, Kontrollgruppe, Katamnese. 


\section{Einleitung}

Die Störung des Sozialverhaltens mit oppositionellem, aufsässigen Verhalten (SSV/OAV, ICD-10 F91.3) ist eine häufige psychische Störung unter Kindern und Jugendlichen (Maughan, Rowe, Messer, Goodman \& Meltzer, 2004). In epidemiologischen und klinischen Stichproben konnten drei Symptomdimensionen der SSV/OAV gefunden werden, welche spezifische Zusammenhänge zu komorbiden und späteren psychischen Störungen zeigten: SSV/OAV-Reizbarkeit als Vorläufer für spätere depressive Erkrankungen und Angststörungen, SSV/OAV-Dickköpfigkeit im Zusammenhang mit komorbiden Aktivitäts- und Aufmerksamkeitsstörungen und SSV/OAV-Boshaftigkeit als Prädiktor für spätere kriminelle Verhaltensweisen (Aebi, Plattner, Winkler Metzke, Bessler \& Steinhausen, 2013; Kolko \& Pardini, 2010; Stringaris \& Goodman, 2009a, 2009b).

Sowohl verhaltensorientierte Behandlungsprogramme für Kinder und Jugendliche (Litschge, Vaughn \& McCrea, 2010; McCart, Priester, Davies \& Azen, 2006) als auch Elterntrainings (McCart et al., 2006; Menting, 2013; Sanders, Kirby, Tellegen \& Day, 2014) konnten mit kleinen bis mittleren Effektstärken das Problemverhalten vermindern. Es zeigte sich zudem, dass bei jüngeren Kindern Elterntrainings doppelt so grosse Effekte hatten wie Kindertherapien alleine, während bei Jugendlichen die Effekte ungefähr gleich gross waren (McCart et al, 2006). Die Behandlung der SSV/OAV sollte indes multimodal erfolgen und somit das problematische Verhalten des Kindes, aber auch bestehende Erziehungsdefizite der Eltern 
berücksichtigen (Knapp et al., 2012; Petermann \& Koglin, 2015; Scotto Rosato et al., 2012; Steiner \& Remsing, 2007). Nur wenige Studien befassten sich mit solchen kombinierten, meist bei jüngeren Kindern angewandten Programmen (z. B. Larson et al., 2009; Webster-Stratton, Reid \& Hammond, 2004). Diese berichteten von mittleren bis grossen Effekten hinsichtlich der Verminderung von aggressivem und regelverletzendem Verhalten.

Das Baghira-Gruppentraining für 8- bis 13-jährige Kinder mit einer SSV/OAV wurde an der Universitätsklinik für Kinder- und Jugendpsychiatrie Zürich entwickelt (Aebi, Perriard, Stiffler Scherrer \& Wettach, 2012). Es soll in Kombination mit dem Triple-P Elterntraining der Interventionsebene vier durchgeführt werden (Ralph \& Sanders, 2006; Sanders, 1999). Letzteres zeigte sich als ein mit mittleren Effektstärken wirksames Verfahren, um die Erziehungskompetenzen von Eltern zu erhöhen und oppositionelles Verhalten von Kindern zu reduzieren (de Graaf, Speetjens, Smit, de Wolff \& Tavecchio, 2008; Sanders et al., 2014). Allerdings können die Ergebnisse vieler Studien zur Behandlung aggressiven Verhaltens von Kindern nicht ohne Einschränkung auf die klinische Grundversorgung übertragen werden (Margraf, 2009). Die meisten empirischen Studien beziehen sich auf Familien, welche sich selber aufgrund von Medieninseraten zugewiesen haben. Diese haben vermutlich eine höhere Behandlungsmotivation und mehr psychosoziale Ressourcen als Familien, welche von anderen Fachpersonen, Behörden und Schulen zur Behandlung überwiesen wurden (Margraf, 2009; Wilson et al., 2012). 
Die vorliegende Pilotstudie hat zum Ziel, in einer klinischpsychiatrischen Institution der Grundversorgung die Wirksamkeit des kognitiv-verhaltenstherapeutischen Baghira-Gruppentrainings in Kombination mit dem Triple-P Elterntraining bei 8- bis 13-jährigen Jungen und Mädchen mit einer diagnostizierten SSV/OAV zu prüfen. Wir gehen davon aus, dass durch die kombinierte Behandlung das externalisierende Verhalten der Kinder - regelverletzendes Verhalten, Aggression, Reizbarkeit, Dickköpfigkeit und Boshaftigkeit - vermindert wird. Ebenso wird eine Verringerung von Impulsivität und Risikoverhalten sowie eine Zunahme der Empathiefähigkeit im Verlauf angenommen.

\section{Methodik}

\subsection{Untersuchungsdesign}

Es handelt sich um eine quasi-experimentelle Untersuchung mit einer Wartelistenkontrollgruppe (WLG). Die Zuweisung zur WLG erfolgte auf Grund des Zeitpunkts der Anmeldung zur Intervention. Wenn diese sechs oder mehr Wochen vor Beginn der Intervention erfolgte, wurde das Kind in die WLG aufgenommen. Die Eltern- und Lehrerangaben zur Behandlungsgruppe (BG) wurden mit denjenigen der WLG verglichen. Veränderungen in Bezug auf externalisierendes Problemverhalten sowie spezifisch in Bezug auf Aggression und regelverletzendes Verhalten wurden zwischen zwei Messzeitpunkten in der WLG (Prä- und Post-Messung) und zwischen drei Messzeitpunkten in der BG erhoben (Prä- und Postmessung sowie Katamnese nach sechs Monaten). Zusätzlich fand eine Einschätzung 
der Behandlungseffekte betreffend die Dimensionen SSV/OAVReizbarkeit, -Dickköpfigkeit und -Boshaftigkeit statt. Eine weitere Analyse in der BG betraf die intraindividuelle Veränderung von Impulsivität, Risikoverhalten und Empathiefähigkeit gemäss Selbstauskunft. Schliesslich wurden die Effekte des Geschlechts, des Intelligenzquotienten (IQ), einer komorbiden Aktivitäts- und Aufmerksamkeitsstörung und des Zuweisungsmodus (Schulbehörden/Jugendeinrichtungen vs. Andere) auf den Behandlungserfolg analysiert.

Die Ethikkommission des Kantons Zürich prüfte und genehmigte die vorliegende Studie.

\subsection{Beschreibung der Stichprobe und des Rekrutierungsablaufs}

Die Studie basiert auf einer konsekutiven Stichprobe von 8- bis 13jährigen Kindern mit der Diagnose einer Störung des Sozialverhaltens mit oppositionellem, aufsässigen Verhalten (SSV/OAV, ICD-10 F91.3), welche zwischen 2007 und 2010 in der Ambulanz der Universitätsklinik für Kinder- und Jugendpsychiatrie Zürich abgeklärt wurden. Ausschlusskriterien waren ein IQ unter 85, das Vorliegen einer komorbiden Störung aus dem schizophrenen Formenkreis, eine schwere affektive Störung, eine neurophysiologische Störung und unzureichende Deutschkenntnisse des Kindes oder der Eltern. Zuletzt wurden auch Familien ausgeschlossen, die mehr als zwei Elterntermine oder mehr als zwei Kindertermine versäumten. 82 Kinder und jeweils mindestens ein Elternteil waren für die Studie verfügbar. Drei Familien verweigerten die Teilnahme an der Studie und elf konnten die Behandlung wegen 
Fehlterminen oder Wegzug nicht wie vorgesehen beenden, was zum Ausschluss aus den Analysen führte. Die Zuweisung zur Intervention erfolgte in 29 Fällen (42.6\%) durch die Schulbehörden oder Jugendeinrichtungen, in 27 Fällen (39.7\%) durch einen Kinderarzt oder sonstigen Therapeuten und in 12 Fällen (17.6\%) durch die Eltern selber. Die Art der Zuweisung war in der WLG und der BG vergleichbar $\left(x^{2}-=1.44, \mathrm{df}=2, \mathrm{p}=0.49\right)$.

Die definitive Stichprobe umfasste 42 Kinder in der BG und 26 Kinder in der WLG. Aus ethischen Gründen wurde auch den 26 Probanden der WLG nach der Wartezeit die gleiche Behandlung wie der BG angeboten. Alle Eltern und Kinder wurden über die Ziele und den Ablauf der Studie aufgeklärt und waren einverstanden, daran teilzunehmen.

\subsection{Interventionen}

Das Baghira-Gruppentraining _ $\quad$ ein kognitivverhaltenstherapeutisches Therapieprogramm für Kinder mit SSV/OAV - basiert auf einem standardisierten Manual (Aebi et al., 2012) und umfasst neun wöchentliche Sitzungen von je 120 Minuten Dauer mit Gruppen von drei bis sechs Kindern. Die Ziele sind eine Verbesserung der sozialen und kommunikativen Fertigkeiten, der emotionalen Kompetenzen, der Ärgerkontrolle sowie der Problemund der Konfliktlösungsfähigkeit. Das Training wurde mit der Absicht entwickelt, die Symptomatik in den Dimensionen SSV/OAVReizbarkeit, -Dickköpfigkeit und -Boshaftigkeit zu vermindern.

Parallel zum Baghira-Gruppentraining erhielten alle Eltern ein standardisiertes Triple-P Erziehungstraining der Interventionsebene 
vier (Positive Parenting Program; Sanders, 1999). Dieses umfasste, abhängig von der Alterstruktur der Therapiegruppe, vier (Turner, Markie-Dadds \& Sanders, 2002) beziehungsweise fünf Sitzungen (Sanders \& Ralph, 2002) von je 120 Minuten Dauer.

\subsection{Datenerhebung}

Demographische Daten, Intelligenz und psychiatrische Diagnosen Anhand der Krankendokumentation der Teilnehmer wurden demographische Daten wie Alter, Geschlecht, Nationalität, Bildung und Berufsstand der Eltern erhoben. In Anlehnung an die schweizerische Gesundheitsbefragung (Swiss Federal Institute for Statistics, 1992) wurde der sozioökonomische Status (SÖS) mit Bezug auf die Bildung und den beruflichen Hintergrund der Eltern definiert: „hoch“ wenn mindestens ein Elternteil einen Gymnasiumoder Hochschulabschluss hatte, selbstständig arbeitete oder Manager mit umfassender Verantwortung war; "mittel“ wenn mindestens ein Elternteil eine abgeschlossene Berufausbildung aufwies oder eine Beschäftigung mit einer entsprechenden Verantwortung inne hatte; „niedrig“ in allen anderen Fällen. Der IQ wurde mit dem Hamburg-Wechsler Intelligenztest für Kinder erhoben (Petermann \& Petermann, 2008; Tewes, Schallberger \& Rossmann, 1999). Fachärzte für Kinder- und Jugendpsychiatrie oder Psychologen mit Masterabschluss erhoben die psychiatrischen Diagnosen der Kinder gemäss den Kriterien der ICD-10.

Externalisierendes Problemverhalten

Die Child Behaviour Checklist (CBCL; Achenbach, 1991a) und die Teacher Rating Form (TRF; Achenbach, 1991b) sind verbreitete, 
reliable und valide Instrumente zur Erfassung von emotionalen Auffälligkeiten und Verhaltensproblemen bei Kindern und Jugendlichen. In der aktuellen Studie wurden gekürzte Versionen der CBCL und der TRF angewendet, welche die externalisierende Skala (CBCL- bzw. TRF-EXT) sowie die CBCL- und TRF-AggressionsSkala als auch die CBCL- und TRF-Regelverletzung-Skala umfassten. Der zeitliche Bezug der CBCL und der TRF wurde von sechs auf zwei Monate gekürzt, um mögliche Behandlungseffekte messen zu können. Die Definition der Subskalen der Dimensionen SSV/OAV-Reizbarkeit, -Dickköpfigkeit und -Boshaftigkeit erfolgte auf der Basis der Items der Skala CBCL-Aggression; diese Subskalen weisen eine gute konstruktive und prädiktive Validität auf (Aebi et al., 2013).

Impulsivität, Risikoverhalten und Empathie gemäss Selbsturteil

Das „Inventar zur Erfassung von Impulsivität, Risikoverhalten und Empathie bei 9- bis 14-jährigen Kindern“ (IVE) ist einer der am häufigsten verwendeten Fragebogen, um selbstberichtetes impulsives Verhalten mit adäquater Reliabilität und Validität zu beurteilen (Luengo, Carrillo-de-la-Pena, Otero \& Romero, 1994; Stadler, Janke \& Schmeck, 2004).

Statistische Datenanalyse

Für die Vergleiche der deskriptiven Daten zwischen der WLG und der BG wurden $X^{2}$-Tests oder t-Tests eingesetzt. Zur Erhebung der intraindividuellen Veränderungen der abhängigen Variablen im Verlauf fanden t-Tests für verbundene Stichproben für die WLG 
(Vergleich zwischen der Prä- und Post-Erhebung) und eine Varianzanalyse für die BG Verwendung (Vergleich zwischen der Prä, Post- und Katamnese-Erhebung). Für die Unterschiede zwischen den Gruppen kamen Kovarianzanalysen mit der abhängigen Variable zum ersten Erhebungszeitpunkt als Kovariate zur Anwendung. Um die Wahrscheinlichkeit einer AlphaFehlerkumulierung durch multiple Testungen zu minimieren, wurde eine Korrektur des Signifikanzniveaus gemäss der Methode von Benjamini und Hochberg (1995) vorgenommen. Die Berechnung der Effektstärken basierte auf Cohens d (Cohen, 1988). Schliesslich wurden univariate lineare Regressionen (LR) mit dem Geschlecht, dem IQ, dem Zuweisungsmodus und dem Vorhandensein einer komorbiden Aktivitäts- und Aufmerksamkeitsstörung als Prädiktoren mit der Veränderung von CBCL- beziehungsweise TRF-EXT während des Behandlungszeitraums als abhängige Variable durchgeführt. Für Probanden, welche zu irgendeinem Zeitpunkt einen fehlenden Itemwert aufwiesen, fand eine Ersetzung gemäss der Methode von Bingham, Stemmler, Petersen und Graber (1998) statt, welche neben dem Stichprobenmittelwert auch den individuellen Mittelwert über den Verlauf in die Berechnung einbezieht.

\section{Ergebnisse}

\subsection{Deskriptive Ergebnisse}

In der gesamten Stichprobe lag die Altersspanne bei 8.0-13.5 Jahre (Durchschnittsalter 10.68, SA=1.36) und das männliche Geschlecht überwog mit $86.7 \%$ deutlich (Tabelle 1). 55.9\% der in der Studie 
berücksichtigten Kinder kamen aus Familien mit mittlerem oder tiefem sozioökonomischen Status. Die häufigste komorbide Störung war die Aktivitäts- und Aufmerksamkeitsstörung (ICD-10 F90.0), welche bei $57.4 \%$ der Probanden diagnostiziert wurde. Eine schwer wiegendere Störung des Sozialverhaltens (ICD-10 F91.0 bis F91.2) wiesen drei Kinder auf (4.4\%), die gleichzeitig auch die Kriterien für SSV/OAV erfüllten. Komorbide emotionale und sonstige Störungen wurden bei weniger als 20\% der Probanden festgestellt. Die Kinder in der WLG unterschieden sich weder betreffend die demographischen Variablen noch hinsichtlich ihres IQ oder anderer komorbider Diagnosen von den Kindern der BG (Tabelle 1). Weiter zeigte sich, dass Probanden in beiden Gruppen bezüglich der PräErhebung von CBCL- und TRF-EXT vergleichbar waren. (CBCL: 21.35 vs. $21.14, t=0.09, d f=66, p=0.93$; TRF: 26.96 vs. $25.43, t=0.51$, $d f=66, p=0.61)$. Gleiches galt für $C B C L-$ und TRF-Aggression (CBCL: 17.04 vs. $16.50, t=0.32, d f=66, p=0.75$; TRF: 23.46 vs. $21.40, t=0.78$, $d f=66, p=0.44)$ sowie für CBCL- und TRF-Regelverletzung (CBCL: 4.31 vs. $4.64, t=-0.49, d f=66, p=0.63$; TRF: 3.50 vs. $4.02, t=-0.91$, $d f=66, p=0.37)$.

\subsection{Veränderung des externalisierenden Verhaltens im Verlauf}

In der BG fanden sich signifikante Mittelwertunterschiede zwischen Prä-, Post- und Katamnese-Erhebung für alle Skalen des CBCL und des TRF, mit Ausnahme der CBCL-Subskala SSV/OAV-Boshaftigkeit (Tabelle 2). Dabei zeigte sich in diesen Subskalen zwischen der Präund der Post-Erhebung sowie auch zwischen der Prä- und der Katamnese-Erhebung durchgehend eine Abnahme des 
Problemverhaltens. In der WLG konnten hingegen keine signifikanten Veränderungen betreffend das von den Eltern beurteilte aggressive und regelverletzende Verhalten festgestellt werden. Dies galt jedoch nicht für die Einschätzung durch die Lehrpersonen: TRFEXT und TRF-Aggression nahmen während der Warteperiode signifikant ab.

3.3 Unterschiede zwischen Wartelistekontroll- und Behandlungsgruppe

Die Vergleiche zwischen der WLG und der BG in Bezug auf erhobene Veränderungsmasse einschliesslich der Effektstärken sind in den beiden rechten Spalten von Tabelle 2 angegeben. Signifikante Unterschiede zwischen WLG und BG wurden betreffend CBCL-EXT, aber nicht TRF-EXT gefunden. Folglich war die Verringerung von CBCL-EXT in der BG ( $m=4.76, S A=6.72)$ signifikant grösser als die Verringerung von CBCL-EXT in der WLG ( $m=1.27, S A=6.89)$, wobei sich eine mittelgrosse Effektstärke von -0.51 ergab. Ähnliche Resultate zeigten sich auch für die Skalen CBCL-Aggression und TRF-Regelverletzung: Zwischen der Prä- und Post-Erhebung sanken in der BG CBCL-Aggression signifikant stärker (CBCL-Aggression: $m=3.62, S A=5.03)$ und TRF-Regelverletzung tendenziell signifikant stärker (TRF-Regelverletzung: $m=0.88, S A=1.76$ ) als in der WLG (CBCL-Aggression: $\quad m=0.85, \quad S A=5.07 ; \quad$ TRF-Regelverletzung: $m=0.00, S A=0.75)$. Keine signifikanten Unterschiede wurden in Bezug auf die Skalen CBCL-Regelverletzung und TRF-Aggression gefunden. Die Abnahme der von den Eltern beurteilten Dimensionen SSV/OAV-Reizbarkeit und SSV/OAV-Dickköpfigkeit wiederum war in 
der BG signifikant grösser (SSV/OAV-Reizbarkeit: $m=0.81, S A=1.57$; SSV/OAV-Dickköpfigkeit: $m=0.90, \quad S A=1.39)$ als in der WLG (SSV/OAV-Reizbarkeit: $m=-0.12, S A=1.42$; SSV/OAV-Dickköpfigkeit: $m=0.15, S A=1.49)$. Dagegen fand sich bei der Dimension SSV/OAVBoshaftigkeit kein signifikanter Unterschied zwischen der BG ( $m=-$ $0.31, S A=1.16)$ und der WLG $(m=0.21, S A=0.90)$.

\subsection{Impulsivität, Risikoverhalten und Empathie im Verlauf}

Die selbst beurteilte Empathiefähigkeit nahm in der BG während der Intervention signifikant zu (Tabelle 3). Hingegen gab es zwischen der Prä- und Post-Messung keine signifikanten Veränderungen der Impulsivität und des Risikoverhaltens.

\subsection{Prädiktoren für Behandlungseffekte in der BG}

Weder das Geschlecht, der IQ noch das Vorhandenseins einer komorbiden Aktivitäts- und Aufmerksamkeitsstörung waren signifikante Prädiktoren für Veränderungen von CBCL-EXT in der BG, wenn mit der Prä-Erhebung von CBCL-EXT als Kontrollvariable eine univariate LR durchgeführt wurde (männliches Geschlecht: $\beta=-$ $0.06, \mathrm{t}=-0.37, \mathrm{p}>0.05$; fremde Nationalität: $\beta=0.13, t=0.88, p>0.05$; IQ: $\quad \beta=0.13, \quad t=-0.80, \quad p>0.05 ; \quad$ komorbide Aktivitäts- und Aufmerksamkeitsstörung: $\beta=0.18, \quad t=-1.13, p>0.05)$. Ebenso war keine der erwähnten Variablen ein signifikanter Prädiktor für TRFEXT in der BG, wenn TRF-EXT aus der Prä-Erhebung als Kontrollvariable einbezogen wurde (männliches Geschlecht: $\beta=-0.27$, $\mathrm{t}=-1.95, \mathrm{p}>0.05 ; \mathrm{IQ}: \beta=-0.13, t=-0.85, p>0.05$; komorbide Aktivitätsund Aufmerksamkeitsstörung: $\beta=-0.19, \quad t=-1.34, \quad p>0.05)$. Ob die 
Zuweisung durch Schulbehörden beziehungsweise Jugendeinrichtungen oder durch Andere erfolgte, hatte keinen Einfluss auf die Veränderung von CBCL-EXT $(\beta=0.10, \quad t=-0.57$, $p>0.05)$ oder TRF-EXT $(\beta=0.02, t=-0.12, p>0.05)$.

\section{Diskussion}

Das Baghira-Gruppentraining (Aebi et al., 2012) in Kombination mit dem Triple-P Elterntraining (Ralph \& Sanders, 2006; Sanders, 1999) erwies sich als wirksam zur Verminderung von aggressiven und regelverletzenden Verhaltensweisen von 8- bis 13-jährigen Kindern mit SSV/OAV, wenn man die Behandlungsgruppe mit einer nicht behandelten Wartelistekontrollgruppe verglich. Insgesamt fanden wir für die Verminderung der von den Eltern beurteilten externalisierenden Verhaltensprobleme ähnliche Effektstärken wie eine frühere Metaanalyse, welche kognitiv-verhaltenstherapeutische Programme zur Behandlung von Aggression und Ärgerproblemen bei Kindern untersuchte (Sukhodolsky, Kassinove \& Gorman, 2004). Über höhere Effektstärken wurde für die Behandlung von Aggression und Trotzverhalten mittels des „Incredible Years Program“ bei 4- bis 8-jährigen Kindern berichtet (Webster-Stratton et al., 2004). Jene Interventionen waren jedoch zeitlich intensiver als die hier verwendete kombinierte Behandlung und umfasste jeweils 22 bis 24 Sitzungen mit den Eltern und 18 Sitzungen mit den Kindern.

Es ist weiter $\mathrm{zu}$ bedenken, dass viele empirische Studien zur Behandlung von aggressiven Verhaltensweisen und zur Förderung erzieherischer Kompetenzen auf Stichproben von jüngeren Kindern 
basieren, deren Eltern selbst motiviert waren und selbständig Hilfe für ihre Erziehungsprobleme suchten (Margraf, 2009; Wilson et al., 2012). Demgegenüber sind Eltern und ältere Kinder, welche von Fachpersonen, Behörden oder Schulen an die kinderpsychiatrische Grundversorgung überwiesen werden, oftmals weniger motiviert und die Verhaltensprobleme der Kinder sind meist schwer wiegender und zeigen sich in unterschiedlichen Kontexten wie Familie, Schule oder Freizeit (Wilson et al. 2012). Die Übertragbarkeit von Forschungsergebnissen in die klinische Praxis ist damit häufig eingeschränkt (Margraf, 2009). Unsere Ergebnisse weisen nun darauf hin, dass eine kombinierte spezifische Gruppenintervention auch in der psychiatrischen Grundversorgung von Kindern wirksam war, die mehrheitlich von Behörden oder Ärzten zur Therapie überwiesen wurden.

Diese kombinierte Intervention ergab Effekte auf verschiedene Verhaltensbereiche des aggressiv-dissozialen Spektrums. Interessanterweise berichteten die Eltern in der vorliegenden Studie von grösseren Behandlungseffekten in Bezug auf aggressives Verhalten als auf regelverletzendes Verhalten. Hingegen berichteten die Lehrpersonen von einer Verminderung von regelverletzenden Verhaltensweisen. Das Problemverhalten eines Kindes kann sich allerdings in verschiedenen Kontexten unterschiedlich darstellen und verändern. Insofern stimmen Einschätzungen von Lehrern und Eltern bezüglich des aggressiven und regelverletzenden Verhaltens häufig nur geringfügig überein (De Los Reyes \& Kazdin, 2005). 
Weiter fanden wir eine signifikante Verminderung der Dimensionen SSV/OAV-Reizbarkeit und SSV/OAV-Dickköpfigkeit. Die Kombination des Kinder- und Elterntrainings mit einem breiten Spektrum von Interventionen konnte somit wie erwartet unterschiedliche Formen aggressiven Verhaltens - zum Beispiel impulsiv-affektive Aggression oder geplante instrumentelle Aggression - positiv verändern. Zudem gibt es Hinweise, dass einige Interventionen wie beabsichtigt auf die Empathiefähigkeit der Kinder wirkten: Die selbst berichtete Empathiefähigkeit der Probanden nahm während der Behandlung zu.

Die Effekte auf das aggressive und regelverletzende Verhalten blieben über einen Zeitraum von sechs Monaten nach Ende der Behandlung stabil. Es ist somit davon auszugehen, dass sowohl die Kinder als auch die Eltern die erlernten Strategien über ein halbes Jahr aufrecht erhalten und nutzbringend einsetzen konnten, sodass das aggressive und regelverletzende Problemverhalten bei einem geringeren Ausmass verblieb.

Es ergaben sich keine Veränderungen der Dimension SSV/OAVBoshaftigkeit sowie der Impulsivität und des Risikoverhaltens gemäss Selbstangaben. Letzteres war unerwartet, da das BaghiraGruppentraining mit Aufgaben zur Verbesserung der emotionalen Selbstkontrolle sowohl die Impulsivität als auch das Risikoverhalten beeinflussen sollte. Die Skala Impulsivität des IVE umfasst jedoch ein breites Spektrum kognitiver und motorischer Impulsivität, während die Interventionen beim Baghira-Gruppentraining indes spezifisch auf eine Verbesserung der Ärgerkontrolle ausgerichtet 
sind. Wenig überraschend war die Dimension SSV/OAVBoshaftigkeit schwierig zu beeinflussen. In vorangehenden Studien wurde die Präsenz von SSV/OAV-Boshaftigkeit als Prädiktor für Behandlungsresistenz identifiziert und deren Zusammenhang mit unemotionalen Persönlichkeitszügen (engl. callous-unemotional traits) aufgezeigt (Kolko \& Pardini, 2010). Das Vorhandensein von unemotionalen Persönlichkeitszügen ist ein Risikofaktor für eine persistierende Verhaltensstörung (Frick, Ray, Thornton \& Kahn, 2014) und bedingt wahrscheinlich intensivere Massnahmen als diejenigen, welche im Rahmen dieser kombinierten Behandlung angeboten werden konnten.

Die Kombination aus Baghira-Gruppentraining und Triple-P Elterntraining war effektiv ungeachtet des IQ, des Geschlechts, der Nationalität sowie einer komorbiden Aktivitäts- und Aufmerksamkeitsstörung. Damit sind die vorliegenden Ergebnisse im Einklang mit früheren Hinweisen, dass Kinder mit SSV/OAV und komorbider Aktivitäts- und Aufmerksamkeitsstörung nicht weniger von kognitiv-verhaltenstherapeutischen Programmen profitieren als solche ohne komorbide Aktivitäts- und Aufmerksamkeitsstörung (Lochman \& Pardini 2008).

Die Ergebnisse der vorliegenden Untersuchung müssen vor dem Hintergrund folgender Limitierungen betrachtet werden: 1) Es handelt sich bei der Wartelistekontrollgruppe ohne Randomisierung um ein mässig robustes Studiendesign. Da keine randomisierte Zuordnung der Probanden zur Behandlungsgruppe (BG) oder Wartelistekontrollgruppe (WLG) möglich war, bleibt unklar, inwieweit 
die Ergebnisse auf bereits zuvor bestehende Unterschiede in den Gruppen zurückgeführt werden könnten. Da die Zuteilung zur BG oder WLG einzig aufgrund des Zeitpunkts der Anmeldung oder Abklärung erfolgte und eine statistische Vorüberprüfung keine Hinweise auf relevante Unterschiede zwischen den beiden Gruppen ergab, erscheint eine Verzerrung allerdings unwahrscheinlich. 2) Trotz der für eine Gruppentherapiestudie eher geringen Fallzahl konnten wir Effekte der kombinierten Intervention aufzeigen. 3) Es fand keine formale Reliabilitätsprüfung der ICD-10 Diagnosen statt. 4) Es waren wegen der Kombination des Baghira-Gruppentrainings mit dem Triple-P Elterntraining keine spezifischen Aussagen über die Effektivität der einzelnen Interventionen möglich. Allerdings empfehlen die geltenden Behandlungsrichtlinien eine solche Kombination von eltern- und kindzentrierten Interventionen (Steiner \& Remsing, 2007).

Als Stärke dieser Studie sind der Einbezug von Eltern-, Lehrer- und Selbstangaben zu mehreren Verlaufszeitpunkten zu erwähnen. Weiter wurden die Dimensionen Reizbarkeit, Dickköpfigkeit und Boshaftigkeit der SSV/OAV einbezogen und es waren deshalb spezifische Aussagen zur Wirksamkeit der Behandlung möglich. Schliesslich basierte die Untersuchung auf einer klinischen Stichprobe und wurde im Rahmen der kinderpsychiatrischen Grundversorgung einer Universitätsklinik durchgeführt. Die Ergebnisse sollten somit auf andere ambulante Kliniken und Versorgungsinstitutionen übertragbar sein. Allerdings sind diese Ergebnisse in einer weiteren Untersuchung mit grösseren Fallzahlen, 
unabhängigen Kind- und Elterninterventionen und einem randomisiert-kontrollierten Forschungsdesign zu überprüfen.

Die vorliegende Pilotstudie zeigt, dass eine kombinierte kognitivverhaltenstherapeutische Kind- und Elternintervention hilfreich ist, um externalisierendes Problemverhalten bei 8- bis 13-jährigen Kindern mit einer SSV/OAV zu reduzieren. Der Effekt war über einen 6-monatigen Katamnesezeitraum hinweg stabil. Diese Ergebnisse unterstützen die kombinierte Anwendung des Baghira-Trainings und des Triple-P Elterntrainings in der ambulanten kinderpsychiatrischen Grundversorgung. 


\section{Literatur}

Achenbach, T. M. (1991a). Manual for the Child Behavior Check List/4-18 and 1991 Profile. Burlington, VT: Department of Psychiatry, University of Vermont.

Achenbach, T. M. (1991b). Manual for the Teacher Report Form and 1991 Profile. Burlington, VT: Department of Psychiatry, University of Vermont.

Aebi, M., Perriard, R., Stiffler Scherrer, B. \& Wettach, R. (2012). Kinder mit oppositionellem und aggressivem Verhalten. Das Baghira Training. Göttingen: Hogrefe.

Aebi, M., Plattner, B., Winkler Metzke, C., Bessler, C. \& Steinhausen, H. C. (2013). Parent- and self-reported dimensions of oppositionality in youth: Construct validity, comorbidity and criminal outcomes in adulthood. Journal of Child Psychology and Psychiatry, 54, 941-949.

Benjamini Y. \& Hochberg Y. (1995). Controlling the false discovery rate: a practical and powerful approach to multiple testing. Journal of the Royal Statistical Society: Series B, 57, 289300.

Bingham, C. R., Stemmler, M., Petersen, A. C. \& Graber, J. (1998). Imputing missing data values in repeated measurement within-subjects designs. Methods of Psychological Research, $3,131-155$.

Cohen, J. (1988). Statistical Power Analysis for the Behavioral Sciences. Hillsdale: Lawrence Erlbaum Association.

de Graaf, I., Speetjens, P., Smit, F., de Wolff, M. \& Tavecchio, L. (2008). Effectiveness of the Triple P Positive Parenting Program on behavioral problems in children: a metaanalysis. Behavior Modification, 32, 714-735.

De Los Reyes, A. \& Kazdin, E. A. (2005). Informant discrepancies in the assessment of childhood psychopathology: A critical review, theoretical framework and recommendations for further study. Psychological Bulletin, 131, 483-509.

Frick, P. J., Ray, J. V., Thornton, L. C. \& Kahn, R. E. (2014). Can callous-unemotional traits enhance the understanding, diagnosis, and treatment of serious conduct problems in children and adolescents? A comprehensive review. Psychological Bulletin, 140, 1-57.

Knapp, P., Chait, A., Pappadopulos, E., Crystal, S., Jensen, P. S. \& Group, T. M. S. (2012). Treatment of maladaptive aggression in youth: CERT guidelines I. Engagement, assessment, and management. Pediatrics, 129, e1562-1576.

Kolko, D. J. \& Pardini, D. A. (2010). ODD dimensions, ADHD, and callous-unemotional traits as predictors of treatment response in children with disruptive behavior disorders. Journal of Abnormal Psychology, 119, 713-725.

Larson, B., Fossum, S., Clifford, G., Drugli, M. B., Handegard, B. H. \& Morch, W.-T. (2009). Treatment of oppositional defiant and 
conduct problems in young Norwegian children. Results of a randomized controlled trial. European Journal of Child and Adolescent Psychiatry, 18, 42-52.

Litschge, C. M., Vaughn M. G. \& McCrea, C. (2010). The Empirical Status of Treatments for Children and Youth With Conduct Problems. An Overview of Meta-Analytic Studies. Research on Social Work Practice, 20(1), 21-35.

Lochman, J. E. \& Pardini, D. A. (2008). Cognitive behavioural therapies. In M. Rutter, D. Bishop, D. Pine, S. Scott, J. Stevenson, E. Taylor et al. (Eds.), Rutter's child and adolescent psychiatry (pp. 1026-1045). Oxford: Blackwell.

Luengo, M. A., Carrillo-de-la-Pena, M. T., Otero, J. M. \& Romero, E. (1994). A short-term longitudinal study of impulsivity and antisocial behavior. Journal of Personality and Social Psychology, 66, 542-548.

Margraf, J. (2009). Übertragbarkeit der Forschungsergebnisse auf die Routinepraxis. In J. Margraf (Hrsg.), Kosten und Nutzen der Psychotherapie (S. 119-125). Heidelberg: Springer.

Maughan, B., Rowe, R., Messer, J., Goodman, R. \& Meltzer, H. (2004). Conduct disorder and oppositional defiant disorder in a national sample: developmental epidemiology. Journal of Child Psychology and Psychiatry, 45, 609-621.

McCart, M. R., Priester, P. E., Davies W. H. \& Azen, R. (2006). Differential effectiveness of behavioral parent-training and cognitiv-behavioral therapy for antisocial youth: a metaanalysis. Journal of Abnormal Child Psychology, 34(4), 527543.

Menting, A. T. A., de Castro, B. O. \& Matthys, W. (2013). Effectiveness of the Incredible Years parent training to modify disruptive and prosocial child behavior: A meta-analytic review. Clinical Psychology Review, 33, 901-913.

Petermann, F. \& Koglin, U. (2015). Therapie aggressiven Verhaltens. In F. Petermann \& U. Koglin (Hrsg.), Aggressive Kinder und Jugendliche (S. 19-31). Heidelberg: Springer.

Petermann, F. \& Petermann, U. (2008). Wechsler Intelligenz Test für Kinder. Göttingen: Hogrefe.

Ralph, A. \& Sanders, M. R. (2006). The 'Teen Triple P' Positive Parenting Program: A preliminary evaluation. Youth Studies Australia, 25, 41-48.

Sanders, M. R. (1999). Triple P-Positive Parenting Program: Towards an empirically validated multilevel parenting and family support strategy for the prevention of behavior and emotional problems in children. Clinical Child and Family Psychology Review, 2, 71-90.

Sanders, M. R., Kirby, J. N., Tellegen, C. L. \& Day, J. J. (2014). The Triple P-Positive Parenting Program: A systematic review and meta-analysis of a multi-level system of parenting support. Clinical Psychological Review, 34, 337-357.

Sanders, M. R. \& Ralph, A. (2002). Practitioner's Manual for Primary Care Teen Triple P. Münster: PAG Institute für Psychology.

Scotto Rosato, N., Correll, C. U., Pappadopulos, E., Chait, A., Crystal, S., Jensen, P. S. et al. (2012). Treatment of 
maladaptive aggression in youth: CERT guidelines II. Treatments and ongoing management. Pediatrics, 129, e1577-1586.

Stadler, C., Janke, W. \& Schmeck, K. (2004). Inventar zur Erfassung von Impulsivität, Risikoverhalten und Empathie. Göttingen: Hogrefe.

Steiner, H. \& Remsing, L. (2007). Practice parameter for the assessment and treatment of children and adolescents with oppositional defiant disorder. Journal of the American Academy of Child and Adolescent Psychiatry, 46, 126-141.

Stringaris, A. \& Goodman, R. (2009a). Longitudinal outcome of youth oppositionality: irritable, headstrong, and hurtful behaviors have distinctive predictions. Journal of the American Academy of Child and Adolescent Psychiatry, 48, 404-412.

Stringaris, A. \& Goodman, R. (2009b). Three dimensions of oppositionality in youth. Journal of Child Psychology and Psychiatry, 50, 216-223.

Sukhodolsky, D. G., Kassinove, H. \& Gorman, B. S. (2004). Cognitive-behavioral therapy for anger in children and adolescents: a meta-analysis. Aggression and Violent Behavior, 9, 247-269.

Swiss Federal Institute for Statistics. (1992). Swiss Health Survey (Schweizerische Gesundheitsbefragung). Retrieved from http://www.bfs.admin.ch/bfs/portal/de/index/infothek/erhebun gen quellen/blank/blank/ess/01.html.

Tewes, U., Schallberger, P. \& Rossmann, U. (1999). HamburgWechsler-Intelligenztest für Kinder III. Bern: Huber.

Turner, K. M. T., Markie-Dadds, C. \& Sanders, M. R. (2002). Manual for Triple $P$ group program. Münster: PAH Institute for Psychology.

Webster-Stratton, C., Reid, M. J. \& Hammond, M. (2004). Treating children with early-onset conduct problems: intervention outcomes for parent, child, and teacher training. Journal of Clinical Child and Adolescent Psychology, 33, 105-124.

Wilson, P., Rush, R., Hussey, S., Puckering, C., Sim, F., Allely, C. S., Doku, P., McConnachie, A. \& Gillberg, C. (2012). How evidence-based is an 'evidence-based parenting program'? A PRISMA systematic review and meta-analysis of Triple P. BMC Medicine, 10, 130.

\footnotetext{
*Korrespondierender Autor:

Dr. phil. Ralph Wettach

Schulgesundheitsdienste

Schulpsychologischer Dienst

Langstrasse 21

$\mathrm{CH}-8004$ Zürich, Schweiz

Tel: +41 444134040

E-Mail: ralph.wettach@zuerich.ch
} 